$>$ Selon de nombreuses études in vitro, la cellule endothéliale cardiovasculaire serait capable de percevoir les variations mécaniques de son environnement immédiat - la circulation sanguine et de les convertir en messages biologiques complexes (remodelage cytosquelettique, régulation de l'expression génique, signalisation intercellulaire). Néanmoins, en raison de la létalité souvent inhérente à l'expérimentation sur le système cardiovasculaire, le rôle de la mécanotransduction des cellules endothéliales in vivo demeure méconnu. De nouvelles expériences de blocage du flux sanguin réalisées chez l'embryon de poisson-zèbre - un modèle animal dont l'oxygénation tissulaire ne dépend pas du système cardiovasculaire - révèlent un rôle essentiel de la mécanotransduction endothéliale dans l'organogenèse, en particulier cardiaque. Ces découvertes suggèrent que certaines cardiomyopathies humaines pourraient résulter d'anomalies de l'hémodynamique et/ou de l'activité transductionnelle des cellules endothéliales, et réaffirment l'importance de la contribution épigénétique dans le contrôle du développement embryonnaire. <

\section{Mécanotransduction des forces hémodynamiques et organogenèse}

Samuel Sidi, Frédéric M. Rosa

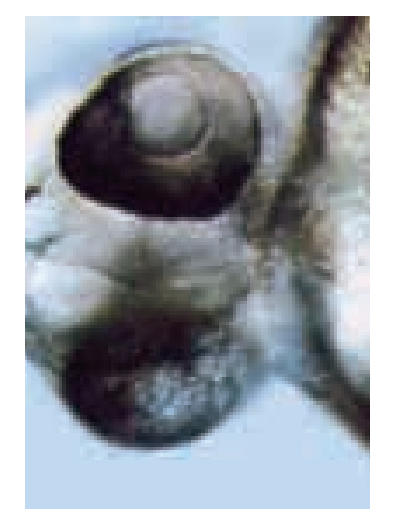

chroniques comme I'hypertension artérielle ou l'artériosclérose [3, 4]. De récentes avancées indiquent que la mécanotransduction endothéliale ne se limite pas au contrôle de la physiologie cardiovasculaire, mais a aussi un impact significatif sur l'organogenèse, en particulier sur le développement cardiaque $[5,6]$. Cette découverte implique notamment que des anomalies de la contribution epigénétique des flux hémodynamiques pourraient être à l'origine de certaines cardiomyopathies. Cette hypothèse, probablement largement sous-estimée jusqu'ici, doit être explorée plus avant.

\section{Mécanotransduction des cellules endothéliales in vitro}

De nombreuses études in vitro réalisées au cours des 20 dernières années militent contre l'idée simpliste selon laquelle les $C \varepsilon$ forment une couche monocellulaire inerte tapissant la surface interne du système cardiovasculaire, dont le rôle se limiterait à celui d'une simple tuyauterie sanguine [3, 4]. Ainsi, l'application d'un flux laminaire à des $C \varepsilon$ en culture induit un réarrangement spectaculaire de leur cytosquelette d'actine (Figure 1) conduisant ces 
cellules à s'allonger et à s'aligner dans la direction du flux $[3,4]$. À cette réponse morphologique s'ajoutent l'activation de NOS3 (nitric oxyde synthase 3), qui catalyse la production du NO (monoxyde d'azote) vasodilatateur, et la stimulation de nombreuses voies de signalisation cellulaires, dont la voie MAP-kinase, aboutissant à la régulation de l'expression de gènes spécifiques $[3,4]$ (Figure 1). Par ailleurs, des régions promotrices sensibles aux stimulus mécaniques ou SSRE (shear stress responsive elements) ont été identifiées, et l'emploi de constructions chimériques SSRE/gène rapporteur démontrent une relation directe entre l'application de forces de flux et la réponse transcriptionnelle [3, 4] (Figure 1). Il apparaît que la réponse génétique des $C \varepsilon$ est très sophistiquée, des groupes de gènes différents étant activés ou réprimés en fonction de la nature du stimulus mécanique, qu'il soit laminaire ou chaotique $[3,4,7]$.

Bien que l'ensemble de ces études ait révolutionné la biologie vasculaire, la signification de l'influence des forces de flux sur la biologie des $C \varepsilon$, et sur le système cardiovasculaire dans son ensemble, reste relativement floue. La circulation sanguine est peu accessible à l'expérimentation et la quantification précise des forces hémodynamiques in vivo reste particulièrement difficile. De plus, les procédures d'expérimentation sur la circulation entraînent des conditions d'hypoxie et d'hyponutrition qui biaisent les analyses phénotypiques. Enfin, les approches génétiques chez la souris sont difficilement envisageables car la perturbation de l'hémodynamique cardiovasculaire chez l'embryon est le plus souvent létale lors de l'embryogenèse.

Récemment, le poisson zèbre (Danio rerio) est apparu comme un modèle de choix pour étudier le développement et la physiologie du système cardiovasculaire in vivo $[8,9]$. L'avantage essentiel de ce modèle est que la survie de l'embryon pendant les grandes étapes de

1 Jekyll code pour l'uridine 5'-diphosphate (UDP)-glucose déshydrogénase, enzyme critique pour la production de l'acide hyaluronique, composant essentiel de la matrice extracellulaire. l'organogenèse ne requiert pas un cœur fonctionnel [59]. Ainsi, même les nombreux mutants cardiaques isolés lors de cribles génétiques survivent aisément jusqu'au stade larvaire (cinq jours post-fécondation) et présentent un comportement locomoteur normal [8,9].

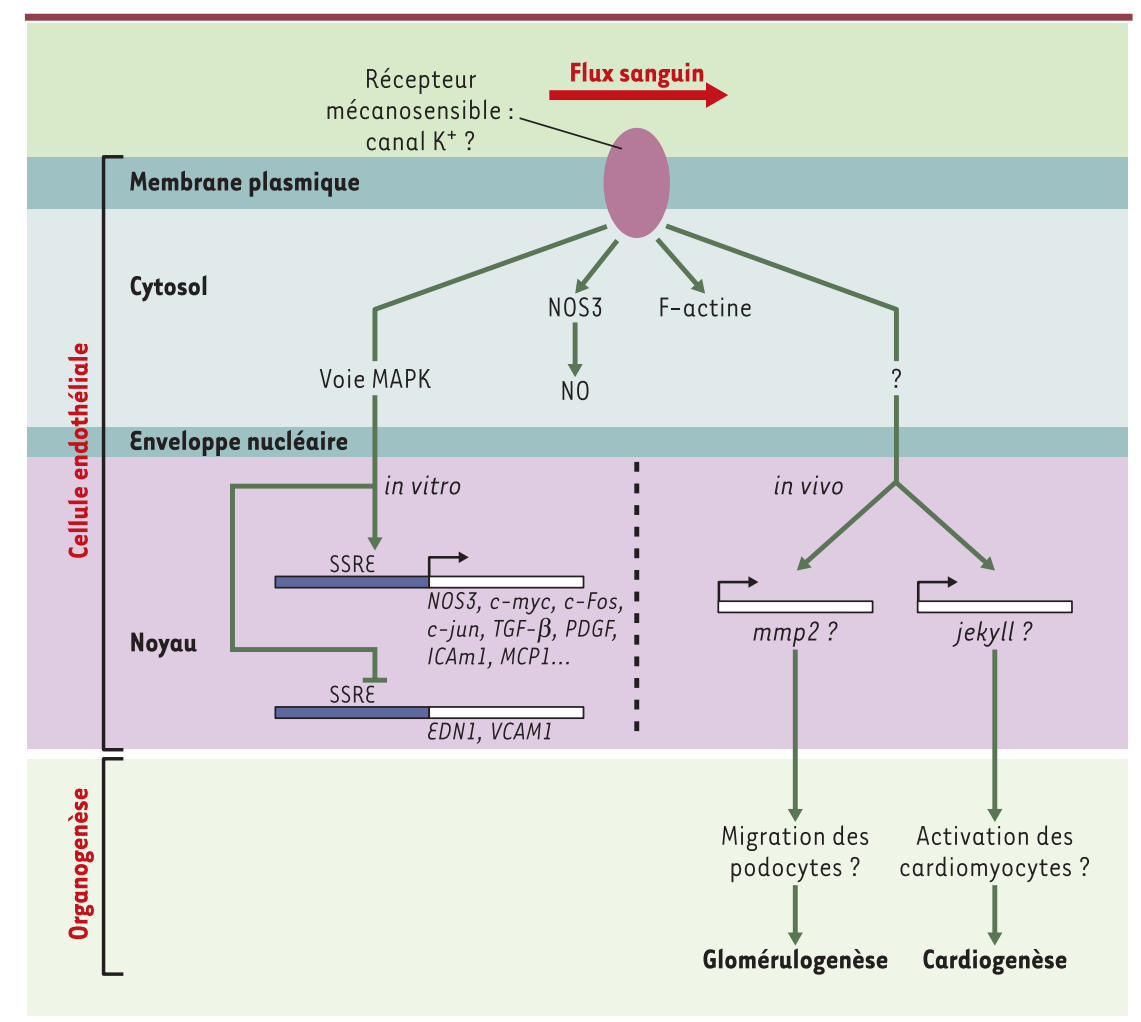

Figure 1. Réponse mécano-sensorielle des cellules endothéliales (cE) aux forces de flux in vitro et in vivo. Une protéine transmembranaire mécanosensible d'identité inconnue, probablement un canal potassique, détecte le stimulus hémodynamique et déclenche diverses réponses dans la $C \varepsilon$. En premier lieu, l'activation de NOS3 (nitric oxide synthase 3), enzyme qui catalyse la formation du monoxyde d'azote (NO), et l'induction d'un réarrangement du cytosquelette d'actine fibrillaire (F-actine), modifient la morphologie cellulaire et contribuent à la vasodilatation. Puis, l'activation de voies de transduction intracellulaires aboutit à la régulation de gènes spécifiques via des régions promotrices mécanosensibles (SSRE). In vitro (à gauche), la voie MAPkinase (MAPK) activerait la transcription de NOS3, de gènes contrôlant le cycle cellulaire ( $c$-myc, c-fos, c-jun), de gènes codant pour des facteurs de croissance comme le TGF $\beta$ (transforming growth factor $\beta$ ) et le PDGF (platelet derived growth factor), des molécules d'adhérence comme ICAMI (intercellular adhesion molecule 1), et des chimiokines comme MCPI (macrophage chemoattractant protein 1). En parallèle, cette même voie réprimerait l'expression de EDNI (endothelinl) codant pour une molécule vasoactive, et de VCAMI (vascular cell adhesion molecule 1) codant pour une protéine d'adhérence (pour revue, voir [3, 4]). Les études de F.C. Serluca et al. [5] et de J.R. Hove et al. [6] laissent supposer que in vivo (à droite), une voie de transduction inconnue, potentiellement la voie MAPK, pourrait activer la transcription de mmp2 (matrix metalloproteinase 2) dans les CE glomérulaires, et de jekyll ${ }^{1}$ dans les CE cardiaques. L'activité collagénase de MMP2 favoriserait la migration en région médiane des précurseurs rénaux (podocytes) et contribuerait ainsi à la glomérulogenèse. Jekyll modifierait la matrice extracellulaire en contact avec les cardiomyocytes et leur permettrait d'activer la transition épitheliomésenchymateuse des $C E$, conduisant au développement des valves cardiaques. 
Ceci s'explique par le fait que les tissus ne nécessitent pas d'apport circulatoire actif en nutriments ou de signalisation hormonale pour se développer, et que l'apport en oxygène par simple diffusion du milieu extérieur vers les tissus est suffisant pour assurer une organogenèse normale [10]. Il est ainsi possible d'étudier la contribution de l'hémodynamique au développement en interférant génétiquement, pharmacologiquement, ou physiquement avec la circulation. À cet avantage s'ajoutent le développement rapide et extra-utérin de l'embryon, ainsi que sa transparence optique, qui facilitent les analyses phénotypiques $[11,12]$. Deux études récentes utilisant le poisson zèbre comme modèle permettent d'ores et déjà d'attribuer un rôle essentiel pour les forces hémodynamiques dans l'organogenèse $[5,6]$.

\section{Flux et développement du rein}

Des anomalies de l'organogenèse rénale sont souvent associées aux anomalies circulatoires sanguines caractéristiques des mutants cardiaques isolés chez le poisson zèbre [13]. Chez ces mutants, la détermination et la différentiation des précurseurs rénaux (podocytes) vers le pronéphros (rein primitif) n'est pourtant pas affectée. En revanche, les podocytes semblent incapables de migrer correctement du mésoderme latéral intermédiaire vers la ligne médiane, où ils fusionnent normalement en région ventrale sous l'aorte dorsale pour former le glomérule. Chez des embryons sauvages, le glomérule entoure une population de CE invasives d'origine aortique [14]. Chez les mutants pickweak (pik), island beat (isl), silent heart (sih) et valentine, les podocytes demeurent en position bilatérale, et les CE migrent anormalement pour finir par entourer les podocytes. Les gènes respectivement affectés chez ces mutants (pik/titin, codant pour une myofibrille cardiaque [15]; isl/cacnalc, codant pour le canal calcique dépendant du potentiel, prédominant des cardiomyocytes [16]; sih/tnnt2, codant pour une troponine cardiaque [17]) ne sont pas exprimés dans les podocytes, suggérant qu'un effet indirect de ces mutations, probablement lié à l'absence de circulation sanguine qu'elles entraînent, est responsable de l'absence de migration des podocytes, et par conséquent, de l'anomalie de développement glomérulaire. $\varepsilon$ n outre, la glomérulogenèse est affectée de façon semblable chez des embryons sauvages dont la circulation est bloquée pharmacologiquement ou par occlusion physique, confirmant que le développement rénal est intimement lié au bon fonctionnement de la circulation sanguine. Le stimulus développemental est-il engendré par le flux sanguin per se (stimulus hémodynamique), ou s'agit-il simplement d'un facteur circulant? Pour trancher entre ces deux hypothèses, Serluca et al. [5] ont remplacé le sang d'embryons sauvages par une simple solution saline isotonique. Le cœur demeure parfaitement fonctionnel, assure la circulation du fluide dans le système cardiovasculaire, et la glomérulogenèse s'effectue normalement. Ceci implique que le stimulus développemental apporté par la circulation n'est pas d'origine cellulaire ou sérique. Ce stimulus pourrait cependant être fourni par l'embryon et véhiculé par la solution saline. Cependant, la rapidité d'induction du phénotype rénal en l'absence de circulation rend cette possibilité improbable et favorise l'hypothèse d'un stimulus de nature mécanique.

Comment les forces hémodynamiques sont-elles reconnues et interprétées par les $C \varepsilon$ pour permettre le déroulement normal de la glomérulogenèse? II semble que le gène mmp2 (matrix metalloproteinase-2), qui code pour une collagénase favorisant la migration cellulaire dans divers tissus [18], et dont l'expression endothéliale est sensible aux contraintes de flux in vitro [19], joue un rôle particulier. En effet, l'expression endothéliale de mmp2 est fortement réduite chez le mutant de circulation isl. De plus, l'injection d'un inhibiteur de MMP2 dans des embryons sauvages abolit la glomérulogenèse, sans affecter la circulation. Serluca et al. [5] concluent que mmp2 agit en réponse à l'action des forces hémodynamiques sur les $C \varepsilon$, et que son activité est nécessaire à la migration et/ou la fusion des podocytes (Figure 1).

\section{Flux et développement cardiaque}

Dans une étude indépendante, Hove et al. [6] ont analysé l'implication des forces de flux sur le développement cardiaque. Se fondant sur de nombreuses études préalables $[3,4,20]$, ces auteurs formulent l'hypothèse que les forces hémodynamiques ont un impact direct sur la cardiogenèse. Afin de tester cette hypothèse in vivo, ils exploitent les avantages expérimentaux offerts par le poisson zèbre, qui leur permettent d'effectuer une quantification détaillée des forces hémodynamiques par diverses techniques d'imagerie. Cette quantification des forces de flux est essentielle ici, car elle permet de tester la pertinence, en regard des études in vitro, des phénotypes observés in vivo.

À 36 h, le primordium cardiaque, visible à faible grossissement, a la forme d'un tube constitué d'une cavité auriculaire reliée à un ventricule, mais est dépourvu de bulbe artériel et de valves. Cette ébauche cardiaque est déjà fonctionnelle et assure un flux sanguin à sens unique dans l'embryon. À quatre jours, le cœur, proche de sa configuration adulte, est constitué de trois chambres communicantes, l'oreillette, le ventricule et le bulbe artériel. Les valves auriculo-ventriculaire et ventriculobulbaire sont également présentes et fonctionnelles à ce stade. Le cycle cardiaque, analysé in vivo par microscopie à transmission à haute vitesse, est identique à celui des vertébrés supérieurs. Brièvement, le sang pénètre dans l'oreillette par le sinus venosus, est ensuite pompé dans le ventricule lors de la diastole, puis éjecté dans le bulbe artériel et dans l'aorte ventrale lors de la systole.

L'étude de la trajectoire des érythrocytes révèle la présence 
d'un flux laminaire ventriculo-bulbaire de grande intensité. De plus, la détermination du volume sanguin intraventriculaire à chaque stade suggère un taux d'éjection voisin de celui du ventricule gauche humain (environ $60 \%$ ). Ce fait, mis en relation avec les dimensions respectives du ventricule et des valves, et avec la durée moyenne de la systole, implique un flux sanguin intracardiaque d'une vitesse d'environ $1,5 \mathrm{~mm} . \mathrm{s}^{-1}$ chez l'embryon et de $1 \mathrm{~cm} . \mathrm{s}^{-1}$ chez la larve. II en résulte que la force de friction excercée sur l'endothélium ou wall shear stress (WSS), est de 2,5 dyn. $\mathrm{cm}^{-2}$ pour le cœur embryonnaire et de 76 dyn. $\mathrm{cm}^{-2}$ pour le cœur larvaire. Ces valeurs de WSS sont remarquablement significatives dans la mesure où une WSS très faible, de l'ordre d'une dyn. $\mathrm{cm}^{-2}$, appliquée à des $C \varepsilon$ en culture, est suffisante pour induire une activation et/ou une répression de l'expression de gènes spécifiques [4]. En outre, la valeur estimée de WSS s'exerçant in vivo sur l'endocarde larvaire excède largement la WSS requise in vitro pour provoquer des réarrangements cytosqueletiques dans les $C \varepsilon$ [4]. Ces données suggèrent que la mécanotransduction de stimulus hémodynamiques par les $C \varepsilon$ pourrait avoir un effet direct, in vivo, sur la cardiogenèse.

Pour tester directement cette hypothèse, J.R. Hove et al. [6] perturbent le flux sanguin en contact direct avec l'endothélium cardiaque embryonnaire. Des billes de verre de $50 \mu \mathrm{m}$ de diamètre sont implantées dans des embryons de $36 \mathrm{~h}$, soit au niveau du sinus venosus, pour empêcher l'arrivée du sang, soit dans le canal ventriculobulbaire, pour bloquer la sortie du sang du cœur vers l'aorte. Des implants témoins (bille à proximité immédiate du sinus venosus mais n'empêchant pas l'accès du sang à l'oreillette) sont insérés en parallèle chez des embryons au même stade. Chez ces derniers, le flux sanguin est normal au cours du développement, et la cardiogenèse s'effectue normalement. En revanche, les spécimens porteurs de billes bloquant le flux développent des malformations cardiaques graves, en particulier une hypotrophie bulbaire, une fusion des sites d'influx et d'efflux sanguins, un positionnement aberrant des cavités cardiaques le long de l'axe antéroposterieur et une absence totale de valves. Ces malformations sont systématiquement corrélées à un reflux sanguin intracardiaque et, en conséquence, à des WSS réduites d'un facteur 10 . II apparaît donc que la réduction du stimulus hémodynamique induite par l'implantation des billes influe significativement sur l'organogenèse cardiaque in vivo.

L'hypothèse de J.R. Hove et al. est d'autant plus convaincante qu'il est a priori possible d'écarter d'autres liens causaux, comme des effets hypoxiques et/ou hyponutritifs [8-10] ou un simple effet des variations de pressions transmurales. En effet, le blocage du flux, qu'il soit exercé au site d'influx (provoquant une diminution de la pression) ou d'efflux (tendant à augmenter la pression), entraîne toujours le même phénotype, et non des malformations différentes. En conclusion, cette étude permet d'envisager un premier lien direct entre mécanotransduction endothéliale des forces hémodynamiques et développement cardiaque, in vivo.

Il est essentiel que cette hypothèse soit confirmée génétique- ment via la production de mutants dont les effets délétères seraient spécifiquement restreints à la cascade mécanotransductionnelle endothéliale, à savoir directement en aval de l'influence du flux sanguin. Il est également indispensable de déterminer quels sont les processus cellulaires affectés par le blocage du flux, et comment leurs perturbations conduisent aux cardiopathies observées.

\section{Réponses cellulaires aux stimulus mécaniques}

De nombreux signaux précoces émanant des CE sont susceptibles d'être activés en réponse directe aux stimulus hémodynamiques. Le signal endothélial conduisant à l'activation des myocardiocytes (cellules sus-jacentes aux $C \varepsilon$ cardiaques) en est un bon exemple. L'activation des myocardiocytes par les CE est indispensable à l'initiation de la transition épithéliomésenchymateuse (TEM), un processus déclenché par les myocardiocytes activés et assurant la transformation des $C \varepsilon$ du canal auriculoventriculaire en « coussins » endocardiques, ébauches des futures valves cardiaques [21]. Le rôle essentiel des CE dans le déclenchement de la TEM a été démontré par analyse du mutant cloche, qui est dépourvu de $C \varepsilon$, et dont les myocardiocytes ne sont jamais activés [22]. Il est possible d'imaginer que chez les embryons implantés de J.R. Hove et al. [6], la faible WSS résultant de la perturbation du flux intracardiaque ne soit pas suffisante pour que les CE émettent le signal activateur vers les myocardiocytes, entraînant à terme l'absence de valves, caractéristique des cardiopathies larvaires observées.

Ce phénotype rappelle l'absence de valves cardiaques du mutant jekyll (jek). jek code une UDP-glucose déshydrogénase nécessaire à la production d'éléments essentiels de la matrice extracellulaire, et semble requis pour l'activation des myocardiocytes [22]. Etant donné que la réduction des forces hémodynamiques dans des cœurs d'embryons de génotype sauvage mime les effets de lésions génétiques dans jek sur la formation des valves, il est possible que jek soit un des gènes activés lors de la réponse mécanosensorielle des $C \varepsilon$ cardiaques, à l'instar de mmp2 dans les $C \varepsilon$ glomérulaires (Figure 1). En conséquence, l'activation des cardiomyocytes serait un processus mécanosensible. Cette hypothèse pourra être testée en comparant le profil d'expression de bmp4 (marqueur de l'activation cardiomyocytaire [22]) dans des cardiomyocytes d'embryons porteurs d'implants bloquant ou non le flux.

Outre l'étude des modalités de la réponse cellulaire endothéliale et la découverte de l'identité des gènes répondant au stimulus mécanique in vivo, une question fascinante concerne les bases moléculaires de la mécanotransduction endothéliale. Dans la plupart des mécanorécepteurs, comme les soies sensorielles de drosophile ou les cellules ciliées de l'oreille interne de vertébré, on considère que l'élément pivot de la machinerie mécanotransductionelle est un canal ionique directement activé par le stimulus mécanique [1, 2]. L'influx d'ions à travers le canal transductionnel dépolarise la membrane et active diverses réponses cellulaires. Les CE possè- 
dent-elles un tel canal ionique? II y a une quinzaine d'années, un courant potassique, spécifiquement activé en réponse aux forces de flux, a été détecté dans des (E en culture [23] (Figure 1). Il est fondamental de déterminer quel est le canal ionique responsable de ce courant et de tester sa sensibilité aux forces de flux.

\section{Conclusions}

Les travaux de J.R. Hove et al. [6] et de F.C. Serluca et al. [5], ainsi qu'une très récente étude chez le poisson zèbre [24], montrent l'importance, jusque-là sous-estimée, du contrôle épigénétique par stimulus mécanique dans l'organogenèse, en particulier cardiaque. Cela permet d'émettre l'hypothèse qu'une part non négligeable des cardiopathies et autres anomalies de l'organogenèse observées chez les mutants du poisson zèbre, et par extrapolation chez l'homme, pourrait résulter d'une altération de ce processus ou de processus similaires. II est important de noter que dans $40 \%$ des cardiomyopathies congénitales humaines, on retrouve des valves anormales [6], ce qui ne manquera pas de motiver les recherches sur la mécanotransduction des forces hémodynamiques dans les prochaines années. $\diamond$

\section{SUMMARY}

Mechanotransduction of hemodynamic forces regulates organogenesis

Endothelial cells $(\varepsilon C)$ of the vertebrate cardiovascular system (CVS) are bona fide, yet enigmatic mechanoreceptors. When cultured in vitro and exposed to fluid forces, $\varepsilon C$ modify their physiological behaviour at the structural, metabolical and gene expression levels in response to the mechanical stimulus. However, as a direct consequence of the hypoxic bias (and often the lethality) that results from blocking blood flow in most animal systems, the in vivo role of $\varepsilon C$ mechanosensation (ECMS) remains poorly understood. The zebrafish has recently emerged as an alternative genetic model for the study of vertebrate development. Its striking ability to survive until larval stages in the absence of blood circulation circumveys the usual caveats that are inherent to CVS research, and offers the exciting opportunity to dissect the function of ECMS in vivo. Two groups have already uncovered an essential role for ECMS in zebrafish organogenesis, particularly in heart morphogenesis. In embryos in which intracardiac blood flow is genetically or physically compromised, several features of the normally developing heart, including valve formation, are specifically disrupted. In addition, impressive imaging studies of zebrafish hemodynamics demonstrate that the shear stress exerted upon the cardiac endothelium is largely in the range of the stimulus that in vitro activates cytoskelettal remodeling and gene expression changes in $\varepsilon$. Hence the cardiac phenotypes observed in vivo may indeed directly result from a lack of ECMS-dependent $\varepsilon C$ activity. These data shed first light on the role of ECMS in vivo. Notably, they also suggest that a number of human congenital cardiomyopathies may arise through abnormal fetal hemodynamics and/or $\varepsilon C$ sensory activity. Finally, these discoveries reinforce the too often neglected role of epigenetic factors (in this case, fluid forces) in the regulation of animal development. $\diamond$

\section{RéFÉRENCES}

1. Gillespie PG, Walker RG. Molecular basis of mechanosensory transduction. Nature 2001; 413: 194-202.

2. Ernstrom GG, Chalfie M. Genetics of sensory mechanotransduction. Annu Rev Genet 2002; 36: 411-53.

3. Topper JN, Gimbrone MA, Jr. Blood flow and vascular gene expression: fluid shear stress as a modulator of endothelial phenotype. Mol Med Today 1999; 5: 40-6.

4. Davies PF. Flow-mediated endothelial mechanotransduction. Physiol Rev 1995; 75: $519-60$.

5. Serluca FC, Drummond IA, Fishman MC. Endothelial signaling in kidney morphogenesis: a role for hemodynamic forces. Curr Biol 2002; 12: 492-7.

6. Hove JR, Koster, RW, Forouhar AS, et al. Intracardiac fluid forces are an essential epigenetic factor for embryonic cardiogenesis. Nature 2003; 421 : 172-7.

7. Topper JN, Cai J, Falb D, Gimbrone MA, Jr. Identification of vascular endothelial genes differentially responsive to fluid mechanical stimuli: cyclooxygenase-2, manganese superoxyde dismutase, and endothelial cell nitric oxide synthase are selectively up-regulated by steady laminar shear stress. Proc Natl Acad Sci USA 1996; 93: 10417-22.

8. Stainier DYR, Fishman MC. The zebrafish as a model system to study cardiovascular development. Trends Cardiovasc Med 1994; 4: 207-12.

9. Stainier DYR. Zebrafish genetics and vertebrate heart formation. Nat Rev Genet $2001 ; 1: 39-48$.

10. Pelster B, Burggren WW. Disruption of hemoglobin oxygen transport does not impact oxygen-dependent physiological processes in developing embryos of zebrafish. Circ Res $1996 ; 79: 358-62$.

11. Haffter $P$, Granato $M$, Brand $M$, et al. The identification of genes with unique and essential functions in the development of the zebrafish, Danio rerio. Development 1996; 123: 1-36.

12. Eisen JS. Zebrafish make a big splash. Cell 1996; 87 : 969-77.

13. Drummond IA, Majumdar A, Hentschel $H$, et al. Early development of the zebrafish pronephros and analysis of mutations affecting pronephric function. Development 1998; 125 : 4655-67.

14. Serluca FC, Fishman MC. Pre-pattern in the pronephric kidney field of zebrafish. Development $2001 ; 128: 2233-41$.

15. Xu X, Meiler SE, Zhong TP, et al. Cardiomyopathy in zebrafish due to a mutation in an alternatively spliced exon of titin. Nat Genet 2002; 30: 205-9.

16. Rottbauer W, Baker K, Wo ZG, et al. Growth and function of the embryonic heart depend on the cardiac-specific L-type calcium channel al subunit. Dev Cell 2001; $1: 265-75$.

17. Sehnert AJ, Huq A, Weinstein BM, et al. Cardiac troponin T is essential in sarcomere assembly and cardiac contractility. Nat Genet 2002; 31: 106-10.

18. Hiraoka N, Allen $\varepsilon$, Apel IJ, et al. Matrix metalloproteinases regulate neovascularization by acting as pericellular fibrinolysins. Cell 1998; 95: 365-77.

19. Bassiouny HS, Song RH, Hong XF, et al. Flow regulation of 72-kD collagenase IV (MMP-2) after experimental arterial injury. Circulation 1998; 98: 157-63.

20. Taber LA. Mechanical aspects of cardiac development. Prog Biophys Mol Biol 1998; 69: $237-55$

21. Bahary N, Zon LI. Endothelium-chicken soup for the endoderm. Science 2001; 294: $530-1$.

22. Walsh EC, Stainier DYR. UDP-glucose deshydrogenase required for cardiac valve formation in zebrafish. Science $2001 ; 293: 1670-3$.

23. Olesen SP, Clapham DE, Davies PF. Hemodynamic shear stress activates a $\mathrm{K}^{+}$current in vascular endothelial cells. Nature 1988; 331: 168-70.

24. Berdougo $\varepsilon$, Coleman $H$, Lee $D H$, et al. Mutation of weak atrium/atrial myosin heavy chain disrupts atrial function and influences ventricular morphogenesis in zebrafish. Development 2003; 130: 6121-9. 